\title{
Tight-binding theory of the spin-orbit coupling in graphene
}

\author{
Sergej Konschuh, Martin Gmitra, and Jaroslav Fabian \\ Institute for Theoretical Physics, University of Regensburg, 93040 Regensburg, Germany \\ (Received 19 July 2010; revised manuscript received 4 October 2010; published 10 December 2010)
}

\begin{abstract}
The spin-orbit coupling in graphene induces spectral gaps at the high-symmetry points. The relevant gap at the $\Gamma$ point is similar to the splitting of the $p$ orbitals in the carbon atom, being roughly $8.5 \mathrm{meV}$. The splitting at the $\mathrm{K}$ point is orders of magnitude smaller. Earlier tight-binding theories indicated the value of this intrinsic gap of $1 \mu \mathrm{eV}$, based on the $\sigma-\pi$ coupling. All-electron first-principles calculations give much higher values, between 25 and $50 \mu \mathrm{eV}$, due to the presence of the orbitals of the $d$ symmetry in the Bloch states at $\mathrm{K}$. A realistic multiband tight-binding model is presented to explain the effects the $d$ orbitals play in the spin-orbit coupling at $\mathrm{K}$. The $\pi-\sigma$ coupling is found irrelevant to the value of the intrinsic spin-orbit-induced gap. On the other hand, the extrinsic spin-orbit coupling (of the Bychkov-Rashba type), appearing in the presence of a transverse electric field, is dominated by the $\pi-\sigma$ hybridization, in agreement with previous theories. Tightbinding parameters are obtained by fitting to first-principles calculations, which also provide qualitative support for the model when considering the trends in the spin-orbit-induced gap in graphene under strain. Finally, an effective single-orbital next-nearest-neighbor hopping model accounting for the spin-orbit effects is derived.
\end{abstract}

DOI: $10.1103 /$ PhysRevB.82.245412

PACS number(s): 31.15.ae, 71.15.Mb, 31.15.aj, 71.90.+q

\section{INTRODUCTION}

Graphene is a two-dimensional allotrope of carbon $^{1}$ that has attracted enormous interest due to both its truly twodimensional nature as well as due to its unique electronic properties originating in the linear energy dispersion at the Fermi level. The spectrum at the $\mathrm{K}$ points is akin to the Dirac cones for massless relativistic particles, causing excitement about the opportunities to test relativistic quantum mechanics in a solid-state material. This ideal picture changes qualitatively when spin-orbit coupling (SOC) is taken into account. Namely, the coupling introduces a gap into the spectrum, giving a mass to the particles. The spectrum is no longer linear. The emergence of the gap moves graphene from the family of semimetals to the one of quantum Hall insulators. ${ }^{2}$ It is ironic that the relativistic (spin-orbit) effects destroy the relativistic appearance of the graphene spectrum. While for many purposes, the spectrum can be still approximated by straight lines, SOC is important when investigating such effects as spin transport, ${ }^{3-6}$ spin relaxation, ${ }^{7-14}$ adatoms on graphene, ${ }^{15}$ or magnetoanisotropies of the predicted magnetic edges. ${ }^{16}$

The question of how large is the spin-orbit-induced gap, the so-called intrinsic gap, at the $\mathrm{K}$ point has been given conflicting answers. The earliest estimates of $200 \mu \mathrm{eV}$ (Ref. 2) were replaced by tight-binding (TB) studies and supporting first-principles results, ${ }^{17,18}$ predicting the gap as small as $1 \mu \mathrm{eV}$. These TB studies considered $s$ and $p$ orbitals. An all-electron first-principles calculation gave a much higher value, of $50 \mu \mathrm{eV},{ }^{19}$ posing an interesting puzzle of what is the actual physics behind the gap. It was recently proved, again from first-principles calculations, that the gap originates from the SOC of (nominally unoccupied) $d$ and higher orbitals. ${ }^{20}$ The predicted gap in that calculation is $24 \mu \mathrm{eV}$. A reasonable estimate, considering the idiosyncrasies of the $a b$ initio codes, is the value of the gap in the range between 25 and $50 \mu \mathrm{eV}$.

The fact that one has to consider $d$ and higher orbitals to get a sizable spin-orbit gap in graphene turns out to has al- ready been known to Slonczewski, ${ }^{21,22}$ who devised a group theoretical argument showing in effect that the SOC of the $p$ orbitals contributes in the second order while that of the $d$ orbitals in the first order. Nice symmetry arguments can be found in Ref. 23. The main point is that without SOC the $p_{z}$ orbitals, that form the relevant states at $\mathrm{K}$, do not hybridize with $p_{x}$ and $p_{y}$. Their hybridization is solely via the spin-orbit interaction. On the other hand, $p_{z}$ do hybridize with $d_{x z}$ and $d_{y z}$ orbitals, forming together the $\pi$ band, as also proven from first-principles work. ${ }^{20}$ Since the $d_{x z}$ and $d_{y z}$ are split by SOC, forming "rotating" orbitals $d_{x z} \pm i d_{y z}$, the gap of the $\pi$ band is linearly proportional to the $d$ splitting.

Graphene has a center of inversion symmetry, making its states doubly (spin) degenerate at a given momentum, even in the presence of SOC. Graphene on a substrate, or under a gate bias, loses this property and the bands further split. This splitting is termed extrinsic, and is akin to the one encountered in semiconductor physics under the name of BychkovRashba splitting or structure inversion asymmetry induced splitting. ${ }^{24,25}$ Only Kramers degeneracy is left, meaning that the energies of the states of opposite spins and momenta are equal. The origin of the extrinsic splitting is the Stark effect, allowing for the $\pi-\sigma$ hybridization, combined with the $p$ orbital SOC. The corresponding TB theory has already been developed; ${ }^{17} d$ orbitals give negligible contribution (of the order of $1 \%$ ), as calculated from first principles ${ }^{20}$ or from our TB theory presented here. The extrinsic gap is about $10 \mu \mathrm{eV}$ for the electric field of $1 \mathrm{~V} / \mathrm{nm}$. This energy scales linearly with the field. A significant enhancement of the extrinsic spin splitting has been reported for graphene placed on a substrate. ${ }^{26-29}$ Large values of the splitting (anything more than $1 \mathrm{meV}$ should be considered as giant here) are likely due to charge transfer between substrate and graphene. When an impurity or an adatom is placed on graphene, the $s p^{3}$ hybridization may distort graphene locally and induce splittings comparable to the values found in zinc-blende semiconductors. ${ }^{15}$

This paper explains the relatively large splitting in the intrinsic graphene from using TB physics. We include the 
relevant $s, p$, and $d$ orbitals and obtain the orbital couplings necessary to account for the splitting by fitting the TB model to first-principles calculations. Our formula for the intrinsic splitting shows that while the contribution from the SOC of the $p$ orbitals increases with increasing lattice constant (decreasing hopping energy), the contribution from the $d$ orbitals decreases. This predicted trend is nicely confirmed from first-principles; as the lattice constant increases, first the splitting decreases, demonstrating the dominance of the $d$ orbitals. After hitting a minimum the splitting increases, being dominated by the SOC of the $p$ orbitals. We also give explicit formulas for the extrinsic splitting, showing that the contributions from the $d$ orbitals are negligible.

For many purposes, such as for investigating spinpolarized transport, magnetoelectric effects, or disorder effects, it is useful to have a simple single-orbital hopping scheme. The functional form of such a hopping Hamiltonian is given by the system symmetries for the specific band region. ${ }^{2}$ We derive such an effective model here by folding down our multiorbital TB scheme to the $\pi$ level, revealing the most relevant hopping paths (that comprise virtual hoppings to other orbitals) and justifying the hopping Hamiltonian from the conventional TB perspective. The resulting spin-dependent next-nearest-neighbor hopping model reproduces well the spin-resolved spectrum of graphene.

The paper is organized as follows. In Sec. II, we introduce the TB model including the relevant $d$ orbitals, and discuss their contribution to the density of states at the Fermi level. In Sec. III A, we turn on the spin-orbit interaction and present the numerical results as well as analytical formulas for the intrinsic SOC induced gap at K. In Sec. III B, we apply a transverse electric field to the graphene sheet and obtain estimates for the $\sigma-\pi$ and $p_{z}-d$ contributions to the extrinsic gap. Finally, in Sec. IV we derive an effective nextnearest-neighbor model for the $\pi$ band in graphene including SOC.

\section{TIGHT-BINDING MODEL}

The hexagonal crystal structure of graphene comprises two atoms in its unit cell. Each set of atoms forms a triangular sublattice conventionally denoted as $A$ and $B$. The Bloch states for each sublattice can be written as a linear combination of localized Wannier functions. In a tightbinding spirit, the Wannier functions are approximated by atomic directed orbitals. These orbitals point along the axes of a chosen coordinate system, in which the three nearest neighbors to an atom at origin are located at

$$
\vec{R}_{i}=\left\{\frac{a}{\sqrt{3}}\left(\begin{array}{l}
0 \\
1
\end{array}\right), \frac{a}{2 \sqrt{3}}\left(\begin{array}{c}
-\sqrt{3} \\
-1
\end{array}\right), \frac{a}{2 \sqrt{3}}\left(\begin{array}{c}
\sqrt{3} \\
-1
\end{array}\right)\right\},
$$

where $a=2.46 \AA$ is the lattice constant. The columns are the two-dimensional coordinates $x$ and $y$, defining the coordinate system.

Considering the nearest neighbors only, the site-dependent TB Hamiltonian reads

$$
H_{\mu, \nu}^{A A}(\vec{k})=H_{\mu, \nu}^{B B}(\vec{k})=\varepsilon_{\mu} \delta_{\mu, \nu},
$$

$$
\begin{gathered}
H_{\mu, \nu}^{A B}(\vec{k})=\sum_{i=1}^{3} e^{i \vec{k} \vec{R}_{i}} t_{\mu, \nu}\left(\vec{n}_{i}\right), \\
H_{\mu, \nu}^{B A}(\vec{k})=\sum_{i=1}^{3} e^{-i \vec{k} \vec{R}_{i}} t_{\mu, \nu}\left(-\vec{n}_{i}\right),
\end{gathered}
$$

where $\mu, \nu$ are the atomic orbitals, $\varepsilon_{\mu}$ are their energies, and $\vec{n}_{i}=\vec{R}_{i} /\left|\vec{R}_{i}\right|$ is the unit vector connecting the neighboring atoms. The hopping matrix elements $t_{\mu, \nu}$, which depend on the bond orientation, are obtained using the two-center SlaterKoster approximation. ${ }^{30}$ The neighboring atomic orbitals are in general not orthogonal. This fact results in the nonzero overlap matrix elements $S_{\mu, \nu}$, which are usually needed to reproduce the electronic spectrum over a wide momentum range. We will give the values for the overlap matrices of the $s$ and $p$ orbitals by fitting to our first-principles calculation. However, for the $\mathrm{K}$ points the overlaps play little role $(<10 \%)$, and we neglect them in our considerations for the SOC.

As the carbon atom has four electrons in the outer shell, one typically resorts to an $8 \times 8$ TB Hamiltonian ${ }^{31}$ for graphene. Without SOC the block-diagonal form of the Hamiltonian contains two different kinds of bands, the $\sigma$ and the $\pi$ bands. The states corresponding to the $\sigma$ bands are responsible for the mechanical (cohesive) properties of graphene while the $\pi$ bands, formed by the $\pi$ bonds between the out-of-plane $p_{z}$ orbitals, are responsible for the unique electronic properties. The conelike dispersion of the $\pi$ bands in the vicinity of the inequivalent $\mathrm{K}$ and $\mathrm{K}^{\prime}$ points, the corners of the hexagonal Brillouin zone, is described by the 2 $\times 2$ Dirac-type Hamiltonian, ${ }^{2}$

$$
H_{0}=\varepsilon_{p}-v_{F}^{0} \hbar\left(\tau \sigma_{x} \kappa_{x}+\sigma_{y} \kappa_{y}\right)
$$

with the Fermi velocity $v_{F}^{0}=\sqrt{3} a V_{p p \pi} /(2 \hbar)$, where $V_{p p \pi}$ is the hopping parameter of the $p_{z}$ orbitals and the wave vector $\vec{\kappa}$ is measured with respect to the Dirac $\mathrm{K}\left(\mathrm{K}^{\prime}\right)$ points, $\vec{k}=\vec{K}\left(\vec{K}^{\prime}\right)$ $+\vec{\kappa}$. The Pauli matrices $\vec{\sigma}$ describe the pseudospin space, such that the eigenstates of $\sigma_{z}$ correspond to the states on the sublattices $A$ and $B$, and $\tau$ accounts for the inequivalent $\vec{K}$ $=(4 \pi / 3 a, 0)(\tau=1)$ and $\vec{K}^{\prime}=(-4 \pi / 3 a, 0)(\tau=-1)$ points. The eigenvalues of $H_{0}$ are given with respect to $\varepsilon_{p}$, the energy of the $p$ orbitals.

The small representation of the $\mathrm{K}$ point, described by the $D_{3 h}$ point group, allows some $d$ orbitals to contribute to the $\pi$-band Bloch states primarily formed by the $p_{z}$ states. ${ }^{21,23} \mathrm{~A}$ natural extension of the TB model in seeking for the additional contributions coming from the $d$ orbitals, is to consider hopping between $p$ and $d$ orbitals. Within the Slater-Koster approximation the $\pi$ bands in graphene are formed by the $p_{z}$, $d_{x z}$, and $d_{y z}$ orbitals, resulting in hopping matrix elements $t_{\mu, \nu}^{\pi}\left(n_{x}, n_{y}\right)$, which are given in Table I. The corresponding Slater-Koster hopping parameters are illustrated in Fig. 1. The TB Hamiltonian, Eq. (2), for the $\pi$ band at the $\mathrm{K}\left(\mathrm{K}^{\prime}\right)$ points has the form 
TABLE I. Slater-Koster hopping matrix elements $t_{\mu, \nu}^{\pi}\left(n_{x}, n_{y}\right)$ of the $\pi$ bands.

\begin{tabular}{lccc}
\hline \hline$\mu \backslash \nu$ & $z$ & $x z$ & $y z$ \\
\hline$z$ & $V_{p p \pi}$ & $n_{x} V_{p d \pi}$ & $n_{y} V_{p d \pi}$ \\
$x z$ & $-n_{x} V_{p d \pi}$ & $n_{x}^{2} V_{d d \pi}+n_{y}^{2} V_{d d \delta}$ & $n_{x} n_{y}\left(V_{d d \pi}-V_{d d \delta}\right)$ \\
$y z$ & $-n_{y} V_{p d \pi}$ & $n_{x} n_{y}\left(V_{d d \pi}-V_{d d \delta}\right)$ & $n_{y}^{2} V_{d d \pi}+n_{x}^{2} V_{d d \delta}$ \\
\hline \hline
\end{tabular}

$$
H=\left(\begin{array}{cccccc}
\varepsilon_{p} & 0 & 0 & 0 & i \tau \alpha & \alpha \\
0 & \varepsilon_{d} & 0 & -i \tau \alpha & \beta & i \tau \beta \\
0 & 0 & \varepsilon_{d} & -\alpha & i \tau \beta & -\beta \\
0 & i \tau \alpha & -\alpha & \varepsilon_{p} & 0 & 0 \\
-i \tau \alpha & \beta & -i \tau \beta & 0 & \varepsilon_{d} & 0 \\
\alpha & -i \tau \beta & -\beta & 0 & 0 & \varepsilon_{d}
\end{array}\right),
$$

given in the basis of $\left(p_{z}^{A}, d_{x z}^{A}, d_{y z}^{A}, p_{z}^{B}, d_{x z}^{B}, d_{y z}^{B}\right)$ orbitals. Here $\alpha=\frac{3}{2} V_{p d \pi}$ and $\beta=\frac{3}{4}\left(V_{d d \delta}-V_{d d \pi}\right)$.

By solving the secular equation $\operatorname{det}(H-\varepsilon I)=0$, where $I$ is the identity matrix, the following two degenerate states can be identified at the Dirac points:

$$
\begin{array}{r}
|1\rangle=\frac{1}{\sqrt{N}}\left[\left|p_{z}^{A}\right\rangle+i \gamma\left(\tau\left|d_{x z}^{B}\right\rangle+i\left|d_{y z}^{B}\right\rangle\right)\right], \\
|2\rangle=\frac{1}{\sqrt{N}}\left[\left|p_{z}^{B}\right\rangle+i \gamma\left(\tau\left|d_{x z}^{A}\right\rangle-i\left|d_{y z}^{A}\right\rangle\right)\right]
\end{array}
$$

with the corresponding normalization $N=1+2 \gamma^{2}$ and $\gamma$ $\approx \alpha /\left(\varepsilon_{d}-\varepsilon_{p}\right)$, assuming $\alpha \ll\left(\varepsilon_{d}-\varepsilon_{p}\right)$. The presence of the $d$ orbitals shifts the eigenenergy of the states, in Eq. (5), with respect to $\varepsilon_{p}$ level, such that $\varepsilon_{1,2} \approx \varepsilon_{p}-2 \gamma^{2}\left(\varepsilon_{d}-\varepsilon_{p}\right)$. It is evident that $\gamma$ and consequently $V_{p d \pi}$ controls the contribution from the $d$ orbital to the $\pi$ bands.

The energy dispersion very close to the $\mathrm{K}$ point remains linear. Here the $p$ - $d$ coupling renormalizes the Fermi velocity
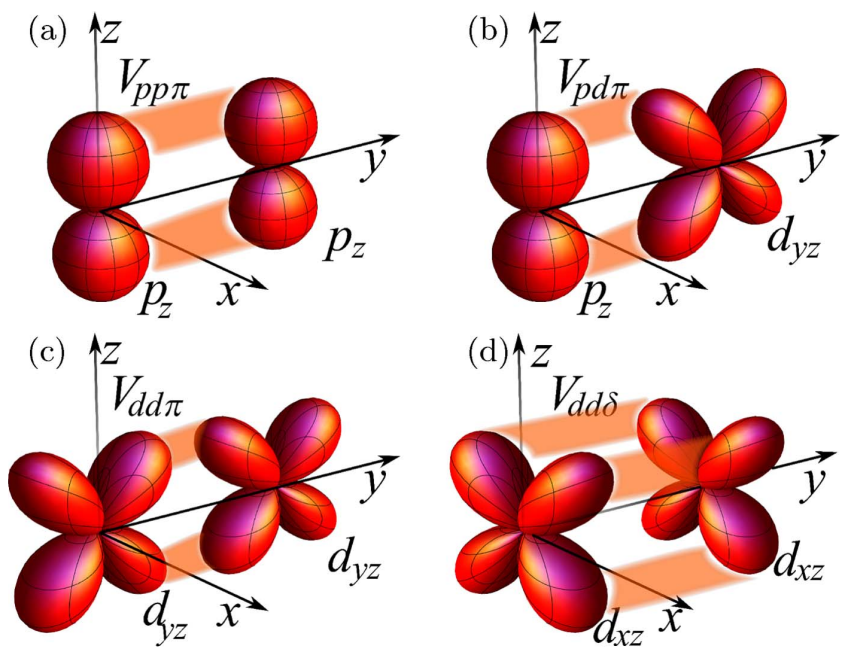

FIG. 1. (Color online) Sketch of the Slater-Koster hopping parameters (a) $V_{p p \pi}$, (b) $V_{p d \pi}$, (c) $V_{d d \pi}$, and (d) $V_{d d \delta}$, needed to calculate the contributions of the $d$ orbitals to the $\pi$ band. according to $v_{F}=v_{F}^{0}\left(1-2 \gamma^{2}\right)$. To obtain a quantitative estimate for $\gamma$ we calculate the density of states (DOS) close to the Fermi level, which can be expressed in terms of $\gamma$,

$$
\mathcal{D}(\varepsilon)=\mathcal{D}_{0}(\varepsilon)\left(1+2 \gamma^{2}\right)^{2},
$$

where $\mathcal{D}_{0}(\varepsilon)=2 \varepsilon / \sqrt{3} \pi V_{p p \pi}^{2}$ is the linear DOS without $d$ orbitals and energy $\varepsilon$ is measured with respect to the Dirac point. In the analytical derivation of Eq. (6) and the energy dispersion, we neglect the hopping between the $d$ orbitals since those contribute only to the states, whose energies are much larger than the Fermi energy. By performing firstprinciples calculations of DOS based on the full-potential augmented plane-wave method, ${ }^{32}$ we can determine the parameter $\gamma$ by calculating the ratio of the DOS slopes close to the Fermi level with and without $d$ orbitals. The ratio is 1.0306 and the slope $\mathcal{D}_{0}^{\prime}(\varepsilon)=0.0392(\mathrm{eV})^{-2}$. The extracted parameter $\gamma \approx 0.0871$ is justifying the assumption $\alpha \ll\left(\varepsilon_{d}\right.$ $\left.-\varepsilon_{p}\right)$. The change in the Fermi velocity of only about $1.5 \%$ is neglected in further calculations. The $d$ orbitals contribute about $3 \%$ to the DOS.

\section{SPIN-ORBIT COUPLING}

\section{A. Intrinsic gap}

SOC connects the electron spin and orbital degrees of freedom. Its major effect comes from the orbits close to the atomic nuclei. Therefore, the crystal potential can be approximated by the spherical atomic potential, which finally gives on-site contribution to the TB Hamiltonian. By averaging the radial degree of freedom the SOC reads

$$
H_{\ell ; \mu, \nu}^{\mathrm{SO}}=\xi_{\ell}\langle\vec{L} \cdot \vec{s}\rangle_{\mu, \nu},
$$

where $\vec{s}$ is the vector of the Pauli matrices representing the real spin and $\vec{L}$ is the angular momentum operator. The matrix element $\langle\cdots\rangle_{\mu, \nu}$ is given in the basis of directed atomic orbitals $\mu, \nu$ and $\xi_{\ell}$ is the angular momentum resolved atomic SOC strength with $\ell=\{s, p, d, \ldots\}$. The matrix elements of the dimensionless SOC operator $\vec{L} \cdot \vec{s}$ for the relevant orbitals in graphene are shown in Table II.

Previous TB calculations ${ }^{7,17,18}$ considered $p$ orbitals only. The SOC of these orbitals removes the block-diagonal form of the Hamiltonian Eq. (2) by coupling the $\pi$ and $\sigma$ bands. This results in the intrinsic SOC splitting of the $\pi$ bands, inducing a gap at the Dirac point of only about $1 \mu \mathrm{eV}$. However, as already discussed by Slonczewski ${ }^{21,22}$ and proven by $a b$ initio calculated by Gmitra et al. ${ }^{20}$ to obtain the spin-orbit splitting at $\mathrm{K}$ in graphene one has to consider $d$ orbitals.

Including $d$ orbitals to the TB Hamiltonian (see Appendix A) and folding down the Hamiltonian using Löwdin transformation (see Appendix B), assuming small spin-orbit coupling and $V_{p d \pi}$ with respect to the energy difference $\varepsilon_{d}-\varepsilon_{p}$, we derive the effective $4 \times 4$ Hamiltonian in the vicinity of the $\mathrm{K}\left(\mathrm{K}^{\prime}\right)$ points,

$$
H_{\text {eff }}=H_{0}+H_{\mathrm{I}} \text {. }
$$

Here $H_{\mathrm{I}}$ is the intrinsic SOC Hamiltonian, having the standard functional form, ${ }^{23}$ 
TABLE II. Matrix elements of the SOC operator $\vec{L} \cdot \vec{s}$ in the basis of $s, p$, and $d$ directed orbitals.

\begin{tabular}{|c|c|c|c|c|c|c|}
\hline Orbital & & $s$ & & $p_{x}$ & $p_{y}$ & $p_{z}$ \\
\hline$s$ & & 0 & & 0 & 0 & 0 \\
\hline$p_{x}$ & & 0 & & 0 & $-i s_{z}$ & $i s_{y}$ \\
\hline$p_{y}$ & & 0 & & $i s_{z}$ & 0 & $-i s_{x}$ \\
\hline$p_{z}$ & & 0 & & $-i s_{y}$ & $i s_{x}$ & 0 \\
\hline Orbital & $d_{x y}$ & & $d_{x^{2}-y^{2}}$ & $d_{x z}$ & $d_{y z}$ & $d_{z^{2}}$ \\
\hline$d_{x y}$ & 0 & & $2 i s_{z}$ & $-i s_{x}$ & $i s_{y}$ & 0 \\
\hline$d_{x^{2}-y^{2}}$ & $-2 i s_{z}$ & & 0 & $i s_{y}$ & $i s_{x}$ & 0 \\
\hline$d_{x z}$ & $i s_{x}$ & & $-i s_{y}$ & 0 & $-i s_{z}$ & $i \sqrt{3} s_{y}$ \\
\hline$d_{y z}$ & $-i s_{y}$ & & $-i s_{x}$ & $i s_{\underline{z}}$ & 임 & $-i \sqrt{3} s_{x}$ \\
\hline$d_{z^{2}}$ & 0 & & 0 & $-i \sqrt{3} s_{y}$ & $i \sqrt{3} s_{x}$ & 0 \\
\hline
\end{tabular}

$$
H_{\mathrm{I}}=\lambda_{\mathrm{I}} \tau \sigma_{z} s_{z} .
$$

The intrinsic spin-orbit induced gap at the $\mathrm{K}\left(\mathrm{K}^{\prime}\right)$ points is twice the intrinsic spin-orbit parameter,

$$
2 \lambda_{\mathrm{I}} \approx \frac{4\left(\varepsilon_{p}-\varepsilon_{s}\right)}{9 V_{s p \sigma}^{2}} \xi_{p}^{2}+\frac{9 V_{p d \pi}^{2}}{\left(\varepsilon_{d}-\varepsilon_{p}\right)^{2}} \xi_{d} .
$$

The first term in Eq. (10) gives a contribution to the intrinsic SOC from the $p$ orbitals, derived previously; ${ }^{7,17,18}$ this contribution is negligible, giving a gap of about $1 \mu \mathrm{eV}$, mainly due to the fact that the SOC of the $p$ orbitals, $\xi_{p}$, appears in the second order. The second term in the above equation is due to the $d$ orbitals and gives a gap of $23 \mu \mathrm{eV}$, as obtained from first-principles. ${ }^{20}$ This term dominates, mainly because the SOC of the $d$ orbitals, $\xi_{d}$, appears in the first order.

To analyze in more detail the contribution from the $d$ orbitals, let us focus on the dependence of the spin-orbit gap on the hopping parameters. In general the hopping parameters decrease with increasing interatomic distance. ${ }^{33}$ The contribution to the gap from the $p$ orbitals is inversely proportional to the square of $V_{s p \sigma}$ and thus should increase with increasing interatomic distance. In Fig. 2, we show the calculations of the intrinsic gap $2 \lambda_{\mathrm{I}}$ as a function of the relative lattice constant $\tilde{a} / a$ stretching. In the absence of $d$ orbitals, the gap increases exponentially and should approach the atomiclike splitting $\Delta=3 \xi_{p} \approx 8.5 \mathrm{meV}$ for an isolated carbon atom. The $d$ orbital contribution is quadratically proportional to $V_{p d \pi}$, thus should vanish for large $\tilde{a} / a$. The resulting decrease and further increase of the gap as the function of $\tilde{a} / a$ is an interplay between the contributions from both the $p$ and $d$ orbitals.

Using the results of the first-principles calculations of the gap in Eq. (10) and the band structure of graphene, we derive the nine parameters of the $s, p$ orbital TB model by fitting the energy spectrum at the high-symmetry points $(\Gamma, \mathrm{K})$ for different artificial lattice constants. The resulting values of the hopping parameters for graphene lattice constant $(\tilde{a} / a$ $=1$ ) are presented in the Table III compared to the previous calculations. ${ }^{31}$ Figure 3 shows the agreement between the band structures along high-symmetry lines obtained by the
TB and first-principles calculation. The energies along the $\mathrm{K} \Gamma$ and $\mathrm{KM}$ lines differs less than $5 \%$ at about $200 \mathrm{meV}$ from $\varepsilon_{F}$, setting the scale for the anisotropy. Taking the hopping parameters in Table III and results of first-principles calculations of $\lambda_{\mathrm{I}}$ we extract the atomic SOC parameters using Eq. (10), where $\xi_{p} \approx 2.8 \mathrm{meV}$. This value coincides with the literature ${ }^{7,17,18}$ taking the different definitions of $\xi_{p}$ into account. Thereby the numerical diagonalization shows a change in this value $<0.01 \%$, that is due to the nonzero overlap parameters. The parameter $\xi_{d} \approx 0.8 \mathrm{meV}$ is obtained, in contrast, using the first-principles calculation results of the DOS and spin-orbit-induced gap using Eqs. (6) and (10). There is no fitting of the energy spectrum like in the $s, p$ case possible, since the needed high-energy states in the conduction bands cannot be identified. The value is linearly sensitive to the $S_{p d \pi}$ overlap. For a reasonable value of $S_{p d \pi}=0.1$ (which is similar to $V_{p p \pi}$, see Table III), the extracted $\xi_{d}$ is within $10 \%$ of what we get for no overlap. This sets the accuracy of the extracted parameters.

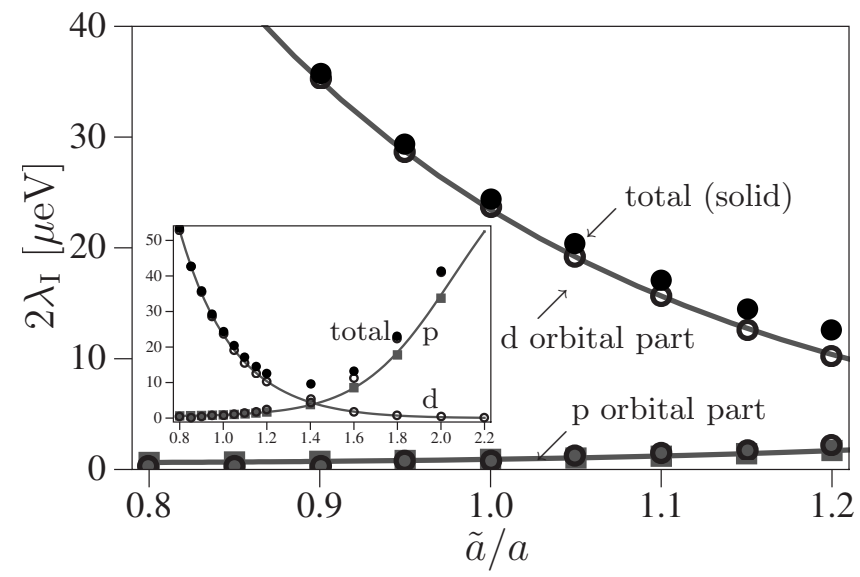

FIG. 2. Results of the first-principles (circles), analytical (solid lines) and numerical (squares) tight-binding calculations of the SOC intrinsic gap in graphene as a function of the artificial lattice constant ratio. Those dependences originate from the hopping parameters. The inset shows the dominance of the $p$ orbitals for larger values of the lattices constant ratio. 
TABLE III. Tight-binding hopping and overlap parameters. The values are obtained by fitting the band structure to the results of the first-principles calculation as the $\Gamma$ and $\mathrm{K}$ points, top row, compared with also presented results given in Ref. 31 .

\begin{tabular}{|c|c|c|c|c|c|}
\hline Parameter & $\varepsilon_{p}-\varepsilon_{s}$ & $V_{s s \sigma}$ & $V_{s p \sigma}$ & $V_{p p \sigma}$ & $V_{p p \pi}$ \\
\hline Energy (eV) & -8.370 & -5.729 & 5.618 & 6.050 & -3.070 \\
\hline Energy $^{a}(\mathrm{eV})$ & -8.868 & -6.769 & 5.580 & 5.037 & -3.033 \\
\hline Parameter & & $S_{s s \sigma}$ & $S_{s p \sigma}$ & $S_{p p \sigma}$ & $S_{p p \pi}$ \\
\hline Value & & 0.102 & -0.171 & -0.377 & 0.070 \\
\hline Value $^{\mathrm{a}}$ & & 0.212 & -0.102 & -0.146 & 0.129 \\
\hline
\end{tabular}

${ }^{\text {aReference } 31 .}$

\section{B. Bychkov-Rashba effect}

An external electric field, that is perpendicular to the graphene plane, breaks spatial inversion symmetry and changes the band structure of graphene. ${ }^{2,20}$ It can originate from a gate voltage or charged impurities in the substrate. The resulting extrinsic SOC leads to spin splitting of the conduction and valence bands due to the Bychkov-Rashba
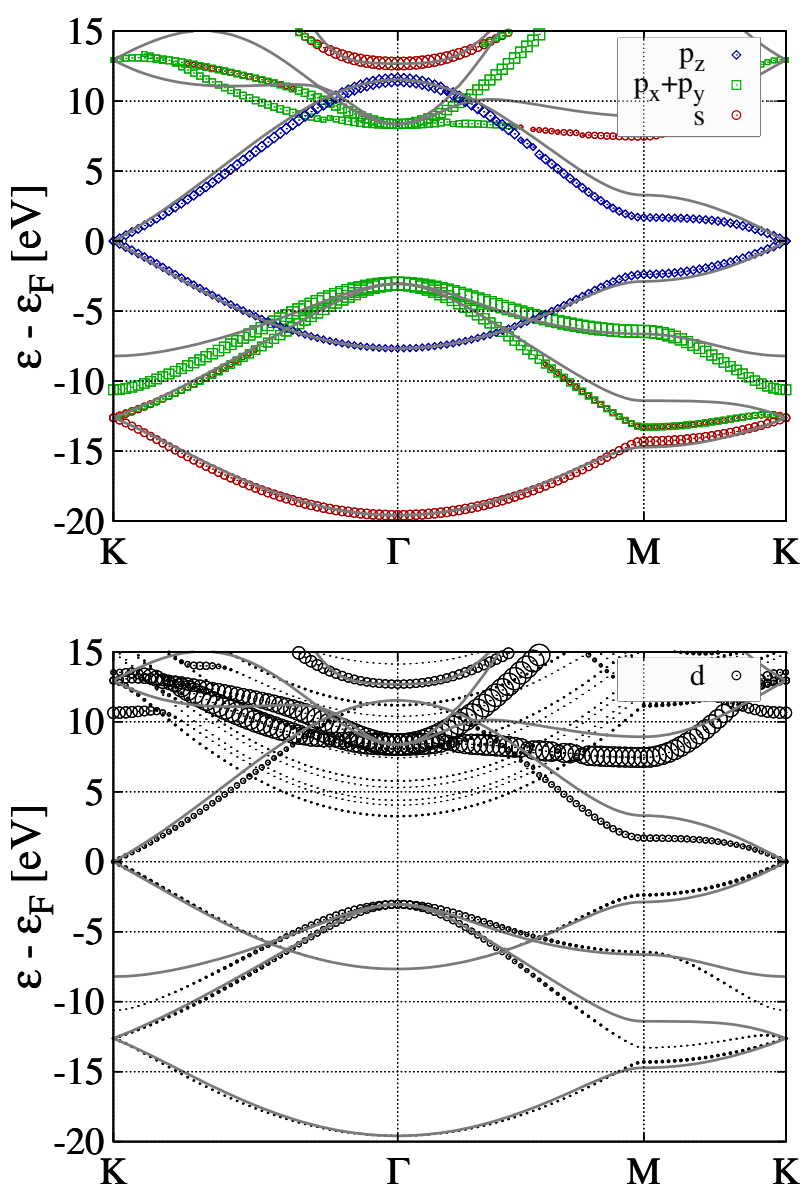

FIG. 3. (Color online) Calculated band structure of graphene obtained from first-principles calculation (symbols) and tightbinding model (solid lines) using the parameters presented in Table III. The size of the symbols reflects the contribution of the orbitals to the corresponding eigenstates. effect. ${ }^{24}$ We investigate this extrinsic SOC by including an electric field into the TB model in the form of the external potential $e E \hat{z}$, which represents an atomic single-particle Stark effect. Due to symmetry, this term allows for the coupling of the $s$ and $p_{z}$ orbitals and the $p_{z}$ and $d_{z^{2}}$ orbitals within the same atom. Nonzero matrix elements of the TB Hamiltonian (see Appendix A) equal $e E z_{s p}$ and $e E z_{p d}$, where $z_{s p}$ and $z_{p d}$ are the expectation values $\left\langle s|\hat{z}| p_{z}\right\rangle$ and $\left\langle p_{z}|\hat{z}| d_{z^{2}}\right\rangle$, respectively, of the operator $\hat{z}$. The electric field leads to a shift of the electron charge density inducing a dipole moment of $0.0134 \mathrm{C} \AA$ in a unit cell for a typical field of $1 \mathrm{~V} / \mathrm{nm} .{ }^{20}$ Since this value is rather tiny we assume that the matrix elements $e E z_{s p}$ and $e E z_{p d}$ are small with respect to $\varepsilon_{p}-\varepsilon_{s}$ and $\varepsilon_{d}-\varepsilon_{p}$, respectively. By folding down the TB Hamiltonian including the atomic SOC and external potential we derive the effective Hamiltonian in the vicinity of the $\mathrm{K}\left(\mathrm{K}^{\prime}\right)$ points,

$$
H_{\text {eff }}=H_{0}+H_{\mathrm{I}}+H_{\mathrm{BR}}
$$

with the Bychkov-Rashba part

$$
H_{\mathrm{BR}}=\lambda_{\mathrm{BR}}\left(\tau \sigma_{x} s_{y}-\sigma_{y} s_{x}\right) .
$$

The Bychkov-Rashba parameter is given by

$$
\lambda_{\mathrm{BR}} \approx \frac{2 e E z_{s p}}{3 V_{s p \sigma}} \xi_{p}+\sqrt{3} \frac{e E z_{p d}}{\left(\varepsilon_{d}-\varepsilon_{p}\right)} \frac{\left.3 V_{p d \pi}\right)}{\left(\varepsilon_{d}-\varepsilon_{p}\right)} \xi_{d} .
$$

The contribution of the $p$ orbitals was already obtained in Ref. 17.

The linear dependence of the Bychkov-Rashba parameter on the electric field $E$ is consistent with the first-principles calculations. ${ }^{20}$ But unlike the intrinsic SOC, the extrinsic contribution due to the $d$ orbitals to $\lambda_{\mathrm{BR}}$ is rather small since the $d$ orbitals contribution is proportional to the product of two small quantities, $V_{p d \pi} /\left(\varepsilon_{d}-\varepsilon_{p}\right)$ and $\xi_{d} /\left(\varepsilon_{d}-\varepsilon_{p}\right)$. According to the first-principles calculations ${ }^{20} \lambda_{\mathrm{BR}}=5 \mu \mathrm{eV}$ for a typical field of $E=1 \mathrm{~V} / \mathrm{nm}$. This is an order of magnitude smaller than the previous predictions by Huertas-Hernando et al. ${ }^{7}$ of the value of $47 \mu \mathrm{eV}$ and Min et al. ${ }^{17}$ of $67 \mu \mathrm{eV}$. The contribution of $d$ orbitals to $\lambda_{\mathrm{BR}}$ is about $1.5 \%$. Comparing the expressions for the Bychkov-Rashba parameters in Eq. (13) with first-principles calculation we obtain $e E z_{s p}$ $\approx 15 \mathrm{meV}$ and the ratio $e E z_{p d} /\left(\varepsilon_{d}-\varepsilon_{p}\right)=0.0003$, which confirms our necessary assumption for the Löwdin transformation used in the derivation of the Eqs. (11) and (13). Figure 4 


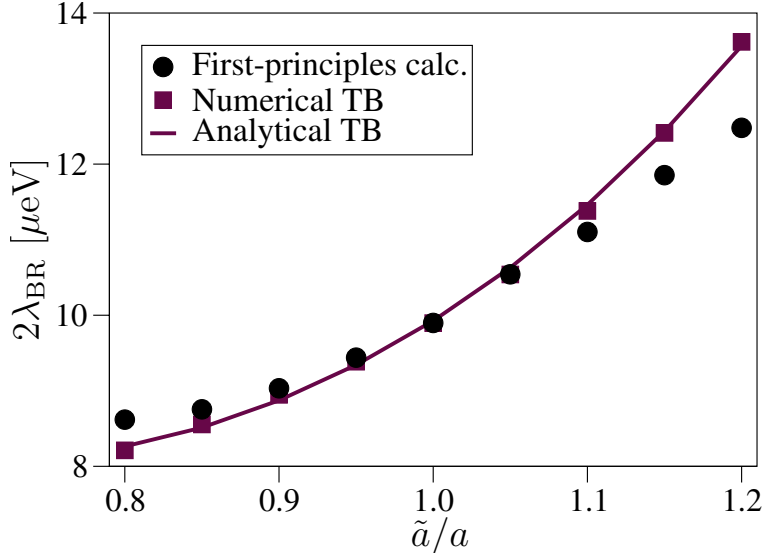

FIG. 4. (Color online) Calculated Bychkov-Rashba constant as a function of the artificial lattice constant ratio: first-principles calculations (circles), numerical diagonalization of the $p$-orbital part of TB Hamiltonian including overlap (squares), and the analytical calculations (solid line). Those dependences on the lattice constant arise from the hopping parameter $V_{s p \sigma}$.

shows the exponential increase of the Bychkov-Rashba parameter $\lambda_{\mathrm{BR}}$ due to the realistic lattice constant stretching, which is controlled by the decay of the hopping parameter $V_{s p \sigma}$ with increasing interatomic distance.

\section{EFFECTIVE NEXT-NEAREST-NEIGHBOR HOPPING}

In the phenomenological description of graphene, the intrinsic and extrinsic SOC originate from the spin-dependent next-nearest-neighbor (nnn) and spin-dependent nearestneighbor (nn) hopping, respectively. ${ }^{2,34}$ The corresponding hopping parameters can be derived from our multiorbital TB model. Doing so, those empirical hopping parameters are expressed by TB parameters, which were introduced in the previous chapters.

The crucial point of our TB model is the absence of spindependent or spin-flipping direct hopping terms. The SOC acts only in the vicinity of the atom core. Hence there is only an effective spin-dependent hopping between two $p_{z}$ orbitals. Such effective hoppings result from a sequence of on-site and nearest-neighbor hoppings within atomic orbitals, the socalled hopping paths. By factoring out the intermediate states (see Appendix C) of the real-space Hamiltonian of noninteracting electrons,

$$
\hat{H}=\sum_{i, j} \sum_{s, s^{\prime}} \sum_{\mu, \nu} c_{\mu, s, i}^{\dagger} T_{\mu, s, i, \nu, s^{\prime}, j} c_{\nu, s^{\prime}, j},
$$

an effective Hamiltonian can be derived. Here the matrix elements $T_{\mu, s, i ; \nu, s^{\prime}, j}$ mark the hopping between the orbitals $\mu$ and $\nu$ with a spin $s$ and $s^{\prime}$, respectively, which belong to the atoms $i$ and $j ; c_{\mu, s, i}^{\dagger}, c_{\nu, s^{\prime}, j}$ are the corresponding creation and annihilation operators. The matrix elements of the neighboring atoms are shown in Table IV using a specific model, that is shown in Fig. 5.

The Hilbert space of the effective Hamilton operator is reduced to that of $p_{z}$ orbitals; we omit these labels in further equations. As predicted, distinct terms, the intrinsic and ex-
TABLE IV. Real-lattice hopping matrix $T$ within the nearestneighbor approximation. The geometrical arrangement is defined in Fig. 5, where the atom labeled by $(p, q)$ belongs to the sublattice $A$. The index $p$ counts the zigzag chains and the index $q$ atoms in a chain. The matrix elements $t_{\mu, \nu}(\vec{n})$ (Ref. 30) contain the nearestneighbor hopping parameters and the on-site matrix elements $\varepsilon_{\mu, \nu}$, the energies of the orbitals and the atomic SOC parameters Eqs. (A3) and (A7).

\begin{tabular}{lccc}
\hline \hline & $p, q-1$ & $p, q$ & $p, q+1$ \\
\hline$p, q-1$ & $\varepsilon_{\mu, \nu}$ & $t_{\mu, \nu}\left(-\vec{n}_{2}\right)$ & 0 \\
$p, q$ & $t_{\mu, \nu}\left(\vec{n}_{2}\right)$ & $\varepsilon_{\mu, \nu}$ & $t_{\mu, \nu}\left(\vec{n}_{3}\right)$ \\
$p, q+1$ & 0 & $t_{\mu, \nu}\left(-\vec{n}_{3}\right)$ & $\varepsilon_{\mu, \nu}$ \\
& $p-1, q-1$ & $p+1, q$ & $p-1, q+1$ \\
\hline$p, q-1$ & $t_{\mu, \nu}\left(-\vec{n}_{1}\right)$ & 0 & 0 \\
$p, q$ & 0 & $t_{\mu, \nu}\left(-\vec{n}_{1}\right)$ & 0 \\
$p, q+1$ & 0 & 0 & $t_{\mu, \nu}\left(-\vec{n}_{1}\right)$ \\
\hline \hline
\end{tabular}

trinsic SOC, appear in the Hamilton operator. The intrinsic SOC operator

$$
\hat{H}_{\mathrm{SO}}=i t_{\mathrm{SO}} \sum_{\langle\langle j, l\rangle\rangle} \sum_{s, s^{\prime}} \zeta_{j, l} c_{s, j}^{\dagger} s_{z} c_{s^{\prime}, l}
$$

contains the nnn hopping with the index $l$ going through all the six next nearest neighbors of the atom $j$. The parameter $\zeta_{j, l}=1(-1)$ for anticlockwise (clockwise) hopping if $j$ is in sublattice $A$; vice versa if $j$ is in $B$. The extrinsic SOC Hamiltonian

$$
\hat{H}_{\mathrm{BR}}=i t_{\mathrm{BR}} \sum_{\langle j, m\rangle} \sum_{s, s^{\prime}} c_{s, j}^{\dagger}\left(\vec{s} \times \vec{n}_{m}\right)_{z} c_{s^{\prime}, m},
$$

appears as hopping within nearest neighbors connected by unit vector $\vec{n}_{m}$. The strengths of the effective hopping are given by the parameters

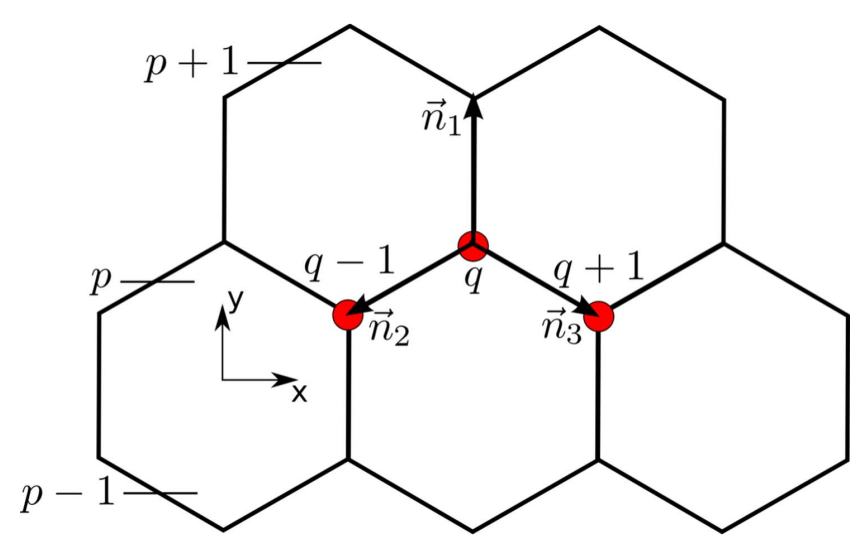

FIG. 5. (Color online) The zig-zag chains in the graphene lattice are defined in the way that the corresponding hopping path goes horizontally, alternating in the direction of the unit vectors $\vec{n}_{2}$ and $\vec{n}_{3}$. The hopping between the chains is possible only in the direction of the unit vector $\vec{n}_{1}$. 


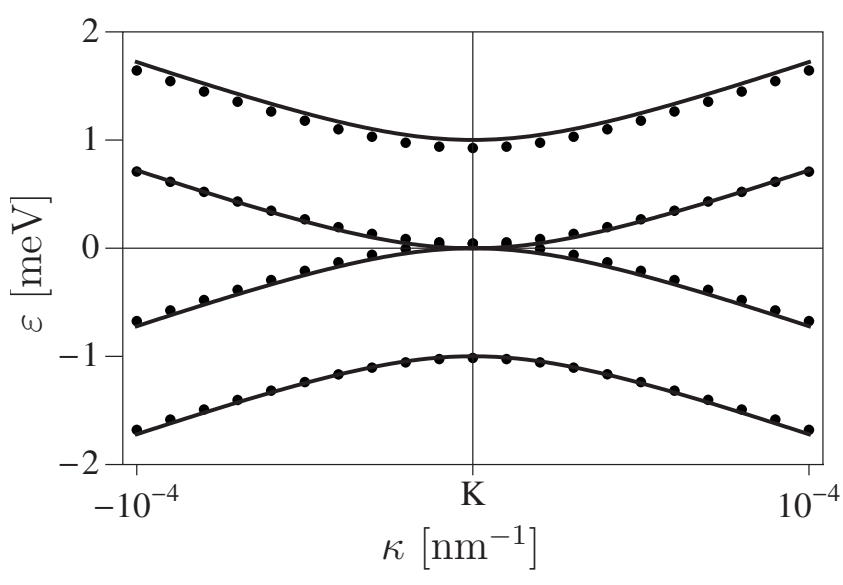

FIG. 6. Calculated spectrum in the vicinity of the K point, plotted along the high-symmetry lines in the $\Gamma-\mathrm{K}-\mathrm{M}$ direction. Circles correspond to the numerical diagonalization of the multiorbital TB Hamiltonian (neglecting the $d$ orbitals which do not play any role here) and solid lines give the eigenenergies of the effective hopping Hamiltonian. The artificially large spin-orbit parameters for this plot are given in the text. The effective hopping model describes well the spin-orbit physics in graphene at the $\mathrm{K}$ point.

$$
t_{\mathrm{SO}}=\frac{\lambda_{\mathrm{I}}}{3 \sqrt{3}}, \quad t_{\mathrm{BR}}=\lambda_{\mathrm{BR}},
$$

which correspond to the SOC parameters given by Eqs. (10) and (13). The effective TB Hamilton matrix Eq. (11) is obtained by Fourier transforming into the $k$ space of the creation and annihilation operators. ${ }^{34}$ The validity of this effective Hamiltonian is again limited, because its eigenenergies $\varepsilon$ are constrained by $\left|\varepsilon-\varepsilon_{p}\right| \ll\left|\varepsilon_{s}-\varepsilon_{p}\right|$ and $\left|\varepsilon-\varepsilon_{p}\right| \ll\left|\varepsilon_{p}-\varepsilon_{d}\right|$ but also $\left\{\xi_{p}, e E z_{s p}\right\} \ll\left|\varepsilon_{s}-\varepsilon_{p}\right|$ and $\xi_{d} \ll\left|\varepsilon_{p}-\varepsilon_{d}\right|$. These conditions limit the wave vector $k$ to the vicinity of the $\mathrm{K}\left(\mathrm{K}^{\prime}\right)$ points; the effective hopping Hamiltonian is valid roughly for $\kappa$ $<2 / a$. Figure 6 shows the band structure of graphene around $\mathrm{K}\left(\mathrm{K}^{\prime}\right)$ points for artificially large values $t_{\mathrm{BR}}=500 \mu \mathrm{eV}$ and $e E z_{s p}=1.5 \mathrm{eV}$. These values are at the limit for the validity of the effective hopping Hamiltonian since higher electric fields would break the linear relation between $t_{\mathrm{BR}}$ and $e E z_{s p}$.

The spin-orbit interaction respects the symmetries of the crystal so no spin-dependent band splitting occurs. Hence it does not couple states of opposite spins, for the spin quantized perpendicular to the sheet. There are two leading hopping paths between two $p_{z}$ orbitals having the same spin, which results in the intrinsic SOC term shown in the Eq. (10). The paths through the states of the $\sigma$ bands, which are constructed by the $s, p_{x}$, and $p_{y}$ orbitals, are shown in Fig. 7. They include two SOC-induced on-site hoppings with the strength $\xi_{p}$, because of the spin flipping. Hence this effect appears in the second order in SOC and the corresponding effective hopping parameter should be negligible small. In Fig. 8, we illustrate the paths through the unfilled $\pi$-band states, the $d_{x z}$ and $d_{y z}$ orbitals. These paths include only one SOC induced on-site spin-conserving hopping with the strength $\xi_{d}$. Therefore this path of hopping is the most responsible for the gap opening, even if the hopping parameter $V_{p d \pi}$ is small compared to the parameter $V_{p p \pi}$. (a)

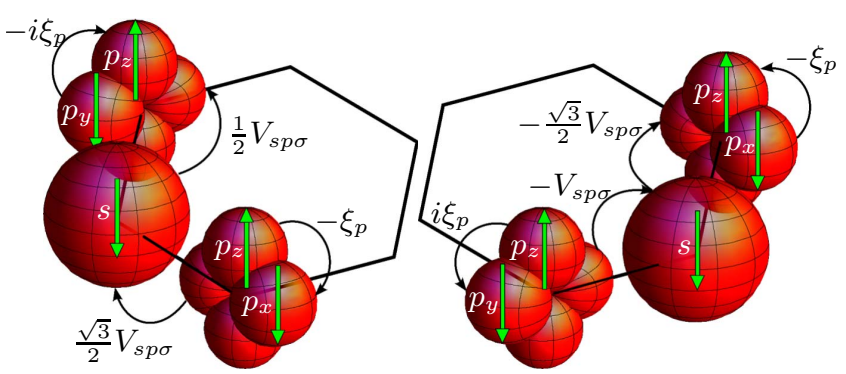

FIG. 7. (Color online) Two of the possible nnn hopping paths through the $s, p$ orbitals, (black) arrows, with a corresponding spin, shown by (yellow) arrows on the orbitals. The opposite sign for the clock-wise (a) and the anti-clock-wise (b) effective hoppings is determined by the signs of the two SOC of the $p$ orbitals.

The Bychkov-Rashba SOC effect originates from the effective nn hopping of two $p_{z}$ orbitals of the opposite spin, appearing in the presence of an applied transverse electric field. The corresponding hopping paths are shown in Fig. 9. The effective nn spin-flipping hopping originates from the coupling to the $\sigma$-band states: a spin-conserving on-site hopping to the $s$ or $d_{z^{2}}$ orbitals and an on-site $s-p$ coupling (via SOC) which flips the spin. The nn hopping takes place between the $s, p$ orbitals and $p, d$ orbitals. In contrast to the intrinsic SOC, the effect of the $d$ orbitals is negligible. Hence the Stark effect coupling of the $p_{z}$ and $d_{z^{2}}$ orbitals is tiny compared to the coupling of $p_{z}$ to the $s$ orbitals. In addition, the SOC coupling of the $d$ orbitals appears an order of magnitude smaller then that of the $p$ orbitals. Finally, the hopping between the $p$ and $d$ orbitals is also smaller then the one between $p$ and $s$ orbitals. Thus the contribution of the sequence of the hopping paths in which the $d$ orbitals take part is quite limited.

Finally we point out that the presented arguments go in hand with the results of our first-principles calculations.

\section{CONCLUSIONS}

We have presented a multiorbital extended TB model for graphene considering $s, p$, and $d$ orbitals relevant when SOC

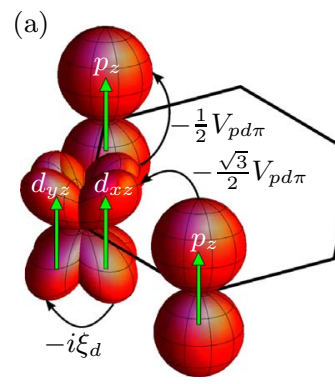

(b)

FIG. 8. (Color online) Two of the possible nnn hopping paths through $d$ orbitals, (black) arrows. Their spin is shown by (yellow) arrows on the orbitals. The opposite sign for clock-wise (a) and anti-clock-wise (b) hopping is given by the opposite sign in the SOC of the $d$ orbitals. 


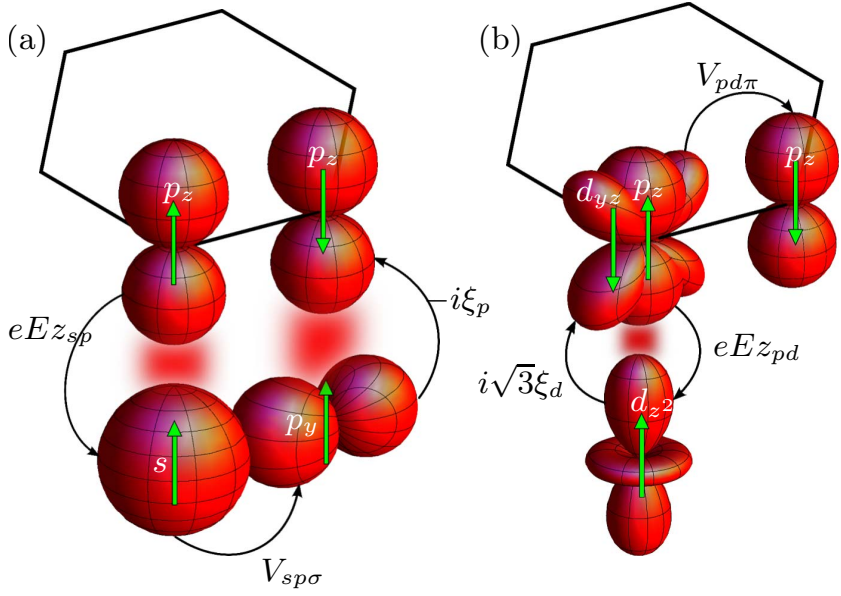

FIG. 9. (Color online) A representative leading hopping path, (black) arrows, which is responsible for the Bychkov-Rashba SOC effect, by coupling states of different spins, illustrated by (yellow) arrows on the orbitals. The effective hopping is between nearest neighbors. (a) The dominant $p$ orbital contribution. (b) The negligible $d$ orbital contribution. For clarity the orbitals of the same atoms are separated vertically, according to their contribution either to the $\sigma$ bands (bottom) or to the $\pi$ bands (top).

is present. Since $d$ orbitals contribute to the $\pi$ bands without SOC, their (atomic) spin-orbit splitting determines the value of the band gap at the $\mathrm{K}\left(\mathrm{K}^{\prime}\right)$ points. This fact is independently confirmed by performing first-principles calculations of the dependence of the SOC induced gap on the lattice constant. We have also derived an effective single-orbital hopping Hamiltonian that captures all the essential spin-orbit physics of itinerant electrons in graphene. With the parameters derived from the multiorbital theory and the insight given by showing the relevant effective hopping paths, such a model should be useful for spin-polarized transport investigations, within the limitations restricting its use close to the $\mathrm{K}\left(\mathrm{K}^{\prime}\right)$ points.

\section{ACKNOWLEDGMENT}

This work was supported by the DFG SPP 1285 and SFB 689.

\section{APPENDIX A}

The extended TB Hamilton matrix can be divided into four blocks,

$$
H=\left(\begin{array}{cc}
H_{\pi} & H_{s} \\
H_{s}^{\dagger} & H_{\sigma}
\end{array}\right) .
$$

The diagonal blocks describe the $\sigma$ bands $\left(H_{\sigma}\right)$ and the $\pi$ bands $\left(H_{\pi}\right)$, which are coupled by SOC, leading to nonzero off-diagonal blocks of the TB Hamiltonian $\left(H_{s}\right)$. The $\sigma$-band block is divided into site-dependent parts due to the two sublattices $(A$ and $B)$ of graphene, which are given in the basis of the $\left\{s, p_{x}, p_{y}, d_{x y}, d_{x^{2}-y^{2}}, d_{z^{2}}\right\}$ atomic orbitals,

$$
H_{\sigma}=\left(\begin{array}{ll}
H_{\sigma}^{A A} & H_{\sigma}^{A B} \\
H_{\sigma}^{B A} & H_{\sigma}^{B B}
\end{array}\right),
$$

where $H_{\sigma}^{A A}=H_{\sigma}^{B B}$ and

$$
H_{\sigma}^{A A}=\left(\begin{array}{cccccc}
\varepsilon_{s} & 0 & 0 & 0 & 0 & 0 \\
0 & \varepsilon_{p} & -i s_{z} \xi_{p} & 0 & 0 & 0 \\
0 & i s_{z} \xi_{p} & \varepsilon_{p} & 0 & 0 & 0 \\
0 & 0 & 0 & \varepsilon_{d} & 2 i s_{z} \xi_{d} & 0 \\
0 & 0 & 0 & -2 i s_{z} \xi_{d} & \varepsilon_{d} & 0 \\
0 & 0 & 0 & 0 & 0 & \varepsilon_{d}
\end{array}\right) .
$$

Here $\varepsilon_{\ell}$ are the energies of the atomic orbitals, $\xi_{\ell}$ the atomic SOC strength of the orbitals with $\ell=\{s, p, d, \ldots\}$ and the Pauli matrices $s_{i}$ with $i=\{x, y, z\}$. The off-diagonal matrices $\left(H_{\sigma}^{A B}\right)^{\dagger}=H_{\sigma}^{B A}$ are presented here at the $\mathrm{K}(\tau=1)$ and the $\mathrm{K}^{\prime}$ $(\tau=-1)$ points

$$
H_{\sigma}^{B A}=\left(\begin{array}{cccccc}
0 & i \tau \alpha_{s} & -\alpha_{s} & i \tau \alpha_{d} & -\alpha_{d} & 0 \\
-i \tau \alpha_{s} & \beta_{p}^{-} & -i \tau \beta_{p}^{-} & -\gamma^{-} & i \tau \gamma^{-} & -i \tau \alpha_{p} \\
\alpha_{s} & -i \tau \beta_{p}^{-} & -\beta_{p}^{-} & i \tau \gamma^{-} & \gamma^{-} & \alpha_{p} \\
i \tau \alpha_{d} & \gamma^{-} & -i \tau \gamma^{-} & -\delta^{-} & i \tau \delta^{-} & i \tau \beta_{d} \\
-\alpha_{d} & -i \tau \gamma^{-} & -\gamma^{-} & i \tau \delta^{-} & \delta^{-} & -\beta_{d} \\
0 & i \tau \alpha_{p} & -\alpha_{p} & i \tau \beta_{d} & -\beta_{d} & 0
\end{array}\right),
$$

end at the $\Gamma$ point

$$
H_{\sigma}^{B A}=\left(\begin{array}{cccccc}
3 V_{s s \sigma} & 0 & 0 & 0 & 0 & -\frac{2}{\sqrt{3}} \alpha_{d} \\
0 & 2 \beta_{p}^{+} & 0 & -2 \gamma^{+} & 0 & 0 \\
0 & 0 & 2 \beta_{p}^{+} & 0 & -2 \gamma^{+} & 0 \\
0 & 2 \gamma^{+} & 0 & 2 \delta^{+} & 0 & 0 \\
0 & 0 & 2 \gamma^{+} & 0 & 2 \delta^{+} & 0 \\
-\frac{2}{\sqrt{3}} \alpha_{d} & 0 & 0 & 0 & 0 & \delta^{0}
\end{array}\right)
$$

defining the parameters

$$
\begin{gathered}
\alpha_{s}=\frac{3}{2} V_{s p \sigma}, \quad \alpha_{p}=\frac{3}{4} V_{p d \sigma}, \quad \alpha_{d}=\frac{3}{4} \sqrt{3} V_{s d \sigma}, \\
\beta_{p}^{ \pm}=\frac{3}{4}\left(V_{p p \pi} \pm V_{p p \sigma}\right), \quad \beta_{d}=\frac{3}{8} \sqrt{3}\left(V_{d d \delta}-V_{d d \sigma}\right), \\
\gamma^{ \pm}=\frac{3}{8}\left(2 V_{p d \pi} \pm \sqrt{3} V_{p d \sigma}\right), \quad \delta^{0}=\frac{3}{4}\left(3 V_{d d \delta}+V_{d d \sigma}\right), \\
\delta^{ \pm}=\frac{3}{16}\left(V_{d d \delta} \pm 4 V_{d d \pi}+3 V_{d d \sigma}\right),
\end{gathered}
$$

which are given by the Slater-Koster hopping parameters $V_{\ell \ell^{\prime} \alpha}$ (Ref. 30) and $\alpha=\{\sigma, \pi, \delta\}$. 
The $\pi$-band block is given in the basis of the $\left\{p_{z}^{A}, p_{z}^{B}, d_{x z}^{A}, d_{y z}^{A}, d_{x z}^{B}, d_{y z}^{B}\right\}$ atomic orbitals. At the $\mathrm{K}\left(\mathrm{K}^{\prime}\right)$ points, it reads as

$$
H_{\pi}=\left(\begin{array}{cc|cccc}
\varepsilon_{p} & 0 & 0 & 0 & i \tau \alpha & \alpha \\
0 & \varepsilon_{p} & i \tau \alpha & -\alpha & 0 & 0 \\
\hline 0 & -i \tau \alpha & \varepsilon_{d} & -i s_{z} \xi_{d} & \beta^{-} & i \tau \beta^{-} \\
0 & -\alpha & i s_{z} \xi_{d} & \varepsilon_{d} & i \tau \beta^{-} & -\beta^{-} \\
-i \tau \alpha & 0 & \beta^{-} & -i \tau \beta^{-} & \varepsilon_{d} & -i s_{z} \xi_{d} \\
\alpha & 0 & -i \tau \beta^{-} & -\beta^{-} & i s_{z} \xi_{d} & \varepsilon_{d},
\end{array}\right),
$$

and at the $\Gamma$ point

$$
H_{\pi}=\left(\begin{array}{cc|cccc}
\varepsilon_{p} & 3 V_{p p \pi} & 0 & 0 & 0 & 0 \\
3 V_{p p \pi} & \varepsilon_{p} & 0 & 0 & 0 & 0 \\
\hline 0 & 0 & \varepsilon_{d} & -i s_{z} \xi_{d} & \beta^{+} & 0 \\
0 & 0 & i s_{z} \xi_{d} & \varepsilon_{d} & 0 & \beta^{+} \\
0 & 0 & \beta^{+} & 0 & \varepsilon_{d} & -i s_{z} \xi_{d} \\
0 & 0 & 0 & \beta^{+} & i s_{z} \xi_{d} & \varepsilon_{d}
\end{array}\right),
$$

where

$$
\alpha=\frac{3}{2} V_{p d \pi}, \quad \beta^{ \pm}=\frac{3}{4}\left(V_{d d \delta} \pm V_{d d \pi}\right),
$$

such that the off-diagonal blocks (separated by lines) contain the hoppings between the $p$ and $d$ orbitals.

Finally the SOC part of the TB Hamiltonian reads as

$$
H_{s}=\left(\begin{array}{cc}
h_{z} & 0 \\
0 & h_{z} \\
h_{x z} & 0 \\
h_{y z} & 0 \\
0 & h_{x z} \\
0 & h_{y z}
\end{array}\right)
$$

where

$$
\begin{aligned}
& h_{z}=\left(\begin{array}{lllll}
e E z_{s p}, & -i s_{y} \xi_{p}, \quad i s_{x} \xi_{p}, & 0, & 0, e E z_{p d}
\end{array}\right), \\
& h_{x z}=\left(0, \quad 0, \quad 0, \quad i s_{x} \xi_{d}, \quad-i s_{y} \xi_{d}, \quad i \sqrt{3} s_{y} \xi_{d}\right),
\end{aligned}
$$

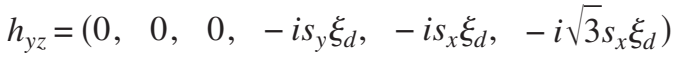

with nonzero Stark-effect matrix elements $e E z_{s p}$ and $e E z_{p d}$. Here $e$ is the electron charge, $E$ the electric field and $z_{s p}, z_{p d}$ are the expectation values of the $\hat{z}$ operator within corresponding orbitals.

\section{APPENDIX B}

To be self-contained, we also present briefly the Löwdin partitioning. The scheme makes use of a unitary anti-
Hermitian operator $S$, such that the transformed Hamiltonian

$$
\tilde{H}=e^{-S} H e^{S} \approx H+[H, S]+\frac{1}{2}[[H, S], S]
$$

has block-diagonal form, with

$$
H=\left(\begin{array}{cc}
H_{0} & T \\
T^{\dagger} & \Delta
\end{array}\right), \quad S=\left(\begin{array}{cc}
0 & M \\
-M^{\dagger} & 0
\end{array}\right),
$$

where $M$ can be obtained iteratively from the equation

$$
T+H_{0} M-M \Delta=0 .
$$

Keeping only second-order terms in $\Delta^{-1}$, the matrix $M$ reads as

$$
M \approx T \Delta^{-1}+H_{0} T \Delta^{-2} .
$$

Inserting this expression into the Eq. (B1), the first element includes the effective Hamiltonian

$$
H_{\mathrm{eff}} \approx H_{0}-T \Delta^{-1} T^{\dagger}-\frac{1}{2}\left\{T \Delta^{-2} T^{\dagger}, H_{0}\right\}
$$

where higher-order terms in $\Delta^{-1}$ are neglected.

\section{APPENDIX C}

To derive the effective hopping energies, Eq. (17), of the next-nearest-neighbor hopping model, with the microscopic SOC parameters specified by Eqs. (10) and (13), and to identify the contributing virtual hopping paths, we start with the secular equation for the multiorbital TB Hamiltonian,

$$
T_{\mu, s, i ; \nu, s^{\prime}, j} c_{\nu, s^{\prime}, j}=\varepsilon c_{\mu, s, i},
$$

where $\mu, \nu$ count the orbitals, $s, s^{\prime}$ the spins, and $i, j$ the atoms. In the nearest-neighbor approximation, the corresponding secular equations of the TB Hamiltonian of the $\pi$ bands, comprising $p_{z}, d_{x z}$, and $d_{y z}$ orbitals, read as

$$
\begin{gathered}
\left(\varepsilon-\varepsilon_{p}\right) c_{z, i}=\sum_{m, \nu} t_{z, \nu}\left(\vec{n}_{m}\right) c_{\nu, m}, \\
\left(\varepsilon-\varepsilon_{d}\right) c_{x z, i}=-i s_{z} \xi_{d} c_{y z, i}+\sum_{m, \nu} t_{x z, \nu}\left(\vec{n}_{m}\right) c_{\nu, m}, \\
\left(\varepsilon-\varepsilon_{d}\right) c_{y z, i}=i s_{z} \xi_{d} c_{x z, i}+\sum_{m, \nu} t_{y z, \nu}\left(\vec{n}_{m}\right) c_{\nu, m} .
\end{gathered}
$$

The spin index is omitted as there are no spin flips; the trace of the spin is kept by $s_{z}$. The label $m$ counts the three nearest neighbors to the atom $i$ and $\nu=\{z, x z, y z\}$ enumerates the $\pi$-band orbitals. The strategy is to eliminate the $d$ orbitals by expressing them via the neighboring $p_{z}$ ones,

$$
\left(\varepsilon_{p}-\varepsilon_{d}\right) c_{x z, m}=\sum_{m^{\prime}}\left[t_{x z, z}\left(-\vec{n}_{m^{\prime}}\right)-i s_{z} t_{y z, z}\left(-\vec{n}_{m^{\prime}}\right) v_{d}\right] c_{z, m^{\prime}},
$$




$$
\left(\varepsilon_{p}-\varepsilon_{d}\right) c_{y z, m}=\sum_{m^{\prime}}\left[t_{y z, z}\left(-\vec{n}_{m^{\prime}}\right)+i s_{z} t_{x z, z}\left(-\vec{n}_{m^{\prime}}\right) v_{d}\right] c_{z, m^{\prime}} .
$$

For the energies $\varepsilon$ in the vicinity of the Dirac point, we use that $\left|\varepsilon-\varepsilon_{d}\right| \approx\left|\varepsilon_{p}-\varepsilon_{d}\right|$. The small parameter $v_{d}$ is given by the ratio $v_{d} \equiv \xi_{d} /\left|\varepsilon_{p}-\varepsilon_{d}\right| \ll 1$. We stay in the linear order in $v_{d}$. The index $m^{\prime}$ now counts the nearest neighbors to the atom $m$; this index thus also includes the original atom $i$. Inserting the above relations into the equation for the $p$ orbitals, Eq. (C2), we obtain the effective second-nearest-neighbor hopping between the $p_{z}$ orbitals via the virtual $d_{x z}$ and $d_{y z}$ orbitals. The first term on the right-hand side of Eqs. (C5) and (C6) results in an energy shift, which was discussed in Sec. II. The second term gives the intrinsic SOC term. The matrix with the elements of the products $t_{z, \mu}\left(\vec{n}_{m}\right) t_{\nu, z}\left(-\vec{n}_{m^{\prime}}\right)$, in which $\mu$ and $\nu$ are the two $d$ orbitals, is

$$
\left(\begin{array}{ll}
n_{m, x} n_{m^{\prime}, x} & n_{m, x} n_{m^{\prime}, y} \\
n_{m, y} n_{m^{\prime}, x} & n_{m, y} n_{m^{\prime}, y}
\end{array}\right) V_{p d \pi}^{2} .
$$

The resulting effective single-orbital hopping model is

$$
\left(\varepsilon-\varepsilon_{p}\right) c_{z, i}=V_{p p \pi} \sum_{m} c_{z, m}+i s_{z} t_{\mathrm{SO}} \sum_{l=1}^{6} \operatorname{rot}(i \rightarrow l) c_{z, l}
$$

where index $l$ denotes the second-nearest neighbors to $i$; $\operatorname{rot}(i \rightarrow l)=1$, if the hopping path from atom $i$ to atom $l$ goes anticlockwise and -1 if clockwise. This direction-sensitive scattering has opposite amplitude for the two (out-of-sheet) spin orientations. The corresponding effective hopping parameter is obtained as $t_{\mathrm{SO}}=\sqrt{3} V_{p d \pi}^{2} \xi_{d} / 2\left(\varepsilon_{p}-\varepsilon_{d}\right)^{2}$.
We now look at the contributions of the $s$ and $p_{x}, p_{y}$ orbitals to the $\pi$ bands, due to the SOC and transverse electric field (the Stark effect). The multiorbital nearest-neighbor TB model gives

$$
\begin{gathered}
\left(\varepsilon-\varepsilon_{p}\right) c_{z, i}=-i s_{y} \xi_{p} c_{x, i}+i s_{x} \xi_{p} c_{y, i}+V_{p p \pi} \sum_{m} c_{z, m}, \\
\left(\varepsilon-\varepsilon_{p}\right) c_{x, i}=i s_{y} \xi_{p} c_{z, i}+\sum_{m, \nu} t_{x, \nu}\left(\vec{n}_{m}\right) c_{\nu, m}, \\
\left(\varepsilon-\varepsilon_{p}\right) c_{y, i}=-i s_{x} \xi_{p} c_{z, i}+\sum_{m, \nu} t_{y, \nu}\left(\vec{n}_{m}\right) c_{\nu, m}, \\
\left(\varepsilon-\varepsilon_{s}\right) c_{s, i}=\xi_{s p} c_{z, i}+\sum_{m, \nu} t_{y, \nu}\left(\vec{n}_{m}\right) c_{\nu, m},
\end{gathered}
$$

where now the index $\nu=\{s, x, y\}$ and $\xi_{s p}=e E z_{s p}$. The SOC connecting the $p_{x}$ and $p_{y}$ orbitals is neglected since it results in higher order contributions in $\xi_{p}$. The strategy to obtain the effective hoppings and virtual hopping paths can be summarized in the following steps: (1) write down the equation for the neighboring $s$ orbitals $c_{s, m}$ assuming $\left|\varepsilon-\varepsilon_{s}\right| \approx\left|\varepsilon_{p}-\varepsilon_{s}\right|$. (2) Eliminate the $s$ orbitals by inserting the equation for $c_{s, m}$ into the equations for $c_{x, i}$ and $c_{y, i}$, neglecting the hopping between the $s$ orbitals. (3) Write down the equations for $c_{x, i}$ and $c_{y, i}$ by neglecting hoppings between $p_{x}$ and $p_{y}$ orbitals. (4) Insert these equations into the one for $c_{z, i}$ assuming $\left|\varepsilon-\varepsilon_{p}\right|$ $\ll V_{s p \sigma}^{2} /\left|\varepsilon_{p}-\varepsilon_{s}\right|$.

The resulting equation for $c_{z, i}$ has the same form as Eq. (C8) except that $t_{\mathrm{SO}}=\sqrt{3}\left(\varepsilon_{p}-\varepsilon_{s}\right) \xi_{p} / 9 V_{s p \sigma}$. In addition, there appears an effective nearest-neighbor hopping parameter $t_{\mathrm{BR}}=2 \xi_{s p} \xi_{p} / 3 V_{s p \sigma}$, in the spin-dependent hopping of the form of Eq. (16), which is due to the combined effect of the transverse electric field and the spin-orbit coupling.
${ }^{1}$ K. S. Novoselov, A. K. Geim, S. V. Morozov, D. Jiang, M. I. Katsnelson, I. V. Grigorieva, S. V. Dubonos, and A. A. Firsov, Nature (London) 438, 197 (2005).; for a review see S. Das Sarma, S. Adam, E. H. Hwang, and E. Rossi, arXiv:1003.4731 (unpublished).

${ }^{2}$ C. L. Kane and E. J. Mele, Phys. Rev. Lett. 95, 226801 (2005).

${ }^{3}$ M. Wimmer, I. Adagideli, S. Berber, D. Tománek, and K. Richter, Phys. Rev. Lett. 100, 177207 (2008).

${ }^{4}$ D. Bercioux and A. De Martino, Phys. Rev. B 81, 165410 (2010).

${ }^{5}$ S.-H. Chen, B. K. Nikolić, and C.-R. Chang, Phys. Rev. B 81, 035428 (2010).

${ }^{6}$ J. Jung and A. H. MacDonald, Phys. Rev. B 81, 195408 (2010).

${ }^{7}$ D. Huertas-Hernando, F. Guinea, and A. Brataas, Phys. Rev. B 74, 155426 (2006).

${ }^{8}$ N. Tombros, C. Jozsa, M. Popinciuc, H. T. Jonkman, and B. J. van Wees, Nature (London) 448, 571 (2007).

${ }^{9}$ K. Pi, W. Han, K. M. McCreary, A. G. Swartz, Y. Li, and R. K. Kawakami, Phys. Rev. Lett. 104, 187201 (2010).

${ }^{10}$ C. Ertler, S. Konschuh, M. Gmitra, and J. Fabian, Phys. Rev. B 80, 041405 (2009).
${ }^{11}$ D. Huertas-Hernando, F. Guinea, and A. Brataas, Phys. Rev. Lett. 103, 146801 (2009).

${ }^{12}$ V. K. Dugaev, E. Y. Sherman, V. I. Ivanov, and J. Barnaś, Phys. Rev. B 80, 081301 (2009).

${ }^{13}$ P. Ingenhoven, J. Z. Bernád, U. Zülicke, and R. Egger, Phys. Rev. B 81, 035421 (2010).

${ }^{14}$ Y. Zhou and M. W. Wu, Phys. Rev. B 82, 085304 (2010).

${ }^{15}$ A. H. Castro Neto and F. Guinea, Phys. Rev. Lett. 103, 026804 (2009).

${ }^{16}$ Y.-W. Son, M. L. Cohen, and S. G. Louie, Nature (London) 444, 347 (2006).

${ }^{17}$ H. Min, J. E. Hill, N. A. Sinitsyn, B. R. Sahu, L. Kleinman, and A. H. MacDonald, Phys. Rev. B 74, 165310 (2006).

${ }^{18}$ Y. Yao, F. Ye, X.-L. Qi, S.-C. Zhang, and Z. Fang, Phys. Rev. B 75, 041401 (2007).

${ }^{19}$ J. C. Boettger and S. B. Trickey, Phys. Rev. B 75, 121402 (2007).

${ }^{20}$ M. Gmitra, S. Konschuh, C. Ertler, C. Ambrosch-Draxl, and J. Fabian, Phys. Rev. B 80, 235431 (2009).

${ }^{21}$ J. C. Slonczewski, Ph.D. thesis, Rutgers University of New Jersey, 1955. 
${ }^{22}$ J. C. Slonczewski and P. R. Weiss, Phys. Rev. 109, 272 (1958).

${ }^{23} \mathrm{~J}$. W. McClure and Y. Yafet, Proceedings of the Fifth Conference on Carbon (Pergamon, New York, 1962), Vol. 1, pp. 22-28.

${ }^{24}$ Y. A. Bychkov and E. I. Rashba, JETP Lett. 39, 78 (1984).

${ }^{25}$ I. Žutić, J. Fabian, and S. Das Sarma, Rev. Mod. Phys. 76, 323 (2004).J. Fabian, A. Matos-Abiague, C. Ertler, P. Stano, and I. Žutić, Acta Phys. Slov. 57, 565 (2007).

${ }^{26}$ E. I. Rashba, Phys. Rev. B 79, 161409 (2009).

${ }^{27}$ I. Gierz, J. Dil, F. Meier, B. Slomski, J. Osterwalder, J. Henk, R. Winkler, C. Ast, and K. Kern, arXiv:1004.1573 (unpublished).

${ }^{28}$ Y. S. Dedkov, M. Fonin, U. Rüdiger, and C. Laubschat, Phys. Rev. Lett. 100, 107602 (2008).

${ }^{29}$ A. Varykhalov, J. Sánchez-Barriga, A. M. Shikin, C. Biswas, E. Vescovo, A. Rybkin, D. Marchenko, and O. Rader, Phys. Rev.
Lett. 101, 157601 (2008).

${ }^{30}$ J. Slater and G. Koster, Phys. Rev. 94, 1498 (1954).

${ }^{31}$ R. Saito, G. Dresselhaus, and M. S. Dresselhaus, Physical Properties of Carbon Nanotubes (Imperial College Press, London, 1998).

${ }^{32}$ P. Blaha, K. Schwarz, G. Madsen, D. Kvasnicka, and J. Luitz, WIEN2-An Augmented Plane Wave Plus Local Orbitals Program for Calculating Crystal Properties (TU Wien, Austria, 2008).

${ }^{33}$ W. A. Harrison, Elementary Electronic Structure (World Scientific, Singapore, 2004).

${ }^{34}$ R. van Gelderen and C. M. Smith, Phys. Rev. B 81, 125435 (2010). 